\title{
A RARE CASE OF PROPTOSIS IN A NEW-BORN CHILD
}

\author{
BY
}

\author{
B. K. Narayana RaO, M.B., M.R.C.S. (Eng.), D.O. (Oxon), \\ Superintendent, Minto Ophthalmic Hospital, Bangalore, India
}

ON October 8, 1925, a new-born Mohammedan female child was brought to hospital at 10 a.m. to be advised "if anything could be done to the baby who had been born without lids to the left eye."

The baby was born at 8 a.m. the same morning. Delivery was normal and was conducted by an experienced local Dhai (unqualified midwife). Soon after birth the left eye was found round, big, prominent, and not covered by lids.

On examination the baby was of full term with no other abnormalities. Right eye was normal. Left eyeball was prominent, proptosed very markedly, was in contact with the brow above, and was without any covering by lids. Cornea was dry and somewhat dull from exposure. Sclera showed slight congestion.

Baby was put on the table, eye washed with boric lotion and the lids fished for with a strabismus hook. After some difficulty both lids were hooked out forward and the eyeball went back with a snap into normal position and the lids covered the eye. Nothing abnormal was felt in the orbit which could have pushed the eyeball to the front. There was only slight oedema of conjunctiva which had disappeared two days later when the child was again seen.

There was evident blepharospasm and the orbicular fibres had gone into a contracted state behind the eveball. Probably either during birth or some time previously in intrauterine life, owing to sudden spasm of the deeper fibres of the orbicularis muscle, or to sudden pushing forward of the eyeball from behind, or by a combination of both causes, the eyeball might have been squeezed out and the lids contracted behind over the optic nerve, sheath, and other contents of the orbit much as in the case of paraphimosis. What the cause of this sudden spasm or forward push was it is difficult to say.

I wished to observe if vision in that eye would show defects later on by this stretching of the optic nerve, but this could not be done as the child died of convulsions when about two months old.

I have looked up the usual text-books on midwifery and a fairly extensive ophthalmic literature and have not found such a case mentioned. 